\title{
Outage Probability of DF IDMA Networks with Best Relay Selection in Weibull Fading
}

\author{
Mehmet Bilim, Nuri Kapucu, Ibrahim Develi \\ Department of Electrical \& Electronics Engineering, Faculty of Engineering, \\ Erciyes University, 38039, Kayseri, Turkey \\ bilim@erciyes.edu.tr; nurikapucu@erciyes.edu.tr; develi@erciyes.edu.tr
}

\begin{abstract}
A dual-hop decode-and-forward (DF) interleave-division multiple access (IDMA) system using best relay selection method in independent Weibull fading channels is addressed. An expression for cumulative distribution function (CDF) is derived by using approximate probability density function (PDF) of end-to-end signal to noise ratio (SNR). Then, the proposed CDF expression is employed to evaluate the outage probability of the considered network. The simulation results are demonstrated to verify the analytical results obtained by using the derived expression, especially at the values of medium and high SNR and to provide further insights.
\end{abstract}

Keywords: Dual-hop, DF IDMA, Best Relay Selection and Weibull.

\section{Introduction}

The best relay selection technique in two-hop and cooperative communication systems is useful for increasing performance and transmission coverage of wireless communication systems. The basic idea behind the best relay selection strategy is to provide similar diversity order compared to the cooperative system and a very simple physical layer to traditional cooperative networks [1]. There are two traditional main relaying protocols such as amplify-and-forward (AF) and decode-and-forward (DF) [2]-[5]. By utilizing advantages of the best relay selection, the performance of cooperative networks were investigated in [6]-[8] for Rayleigh environments and in [9] and [10] for Nakagami-m links. In [6] and [7], the error performance and outage probability of adaptive DF in the cooperative diversity systems using the best relay selection method were proposed in independent non-identical Rayleigh links, respectively. These studies presented a analytical model to analyse the error performance and the closed-form expression for the outage probability. The authors in [8] examined the AF and DF cooperative networks using $N$ th best relay selection technique for Raylegih links. They derived closed-form expressions for the error probability and outage probability in their study. In [9], the error performance of opportunistic DF relaying over Nakagami- $m$ fading channels was investigated and the moment generating function (MGF) expression by using Lauricella hypergeometric function for their scheme was obtained. In another work in [10], the authors presented the outage probability of a two-way relaying with AF protocol using relay selection in Nakagami- $m$ links and derived a closed-form approximation. They concluded that the coding gain and outage performance of the considered system can easily evaluated by employing the derived outage probability expression.

Some important publications have focused on the two-hop DF/AF relaying networks using best relay selection [1113]. The closed-form expressions for the cumulative distribution function (CDF) and the probability density function (PDF) of the total instantaneous signal to noise ratio (SNR) of two-hop systems with AF protocol using relay selection were studied in [11]. In [12], a multiple-input multiple-output (MIMO) system with two-hop relay selection protocol is considered. They investigated the outage probability of the considered system, asymptotic and exact outage expressions are also proposed. The optimum combining performance of AF relaying networks is analysed employing an approximation at the destination [13]. However, all the studies in [6-13] are based on the cooperative or dual-hop transmission without multi-user communication systems.

To promote transmission performance in multi-user communication systems, interleave-division multiple access (IDMA) systems was introduced by employing low-complexity receiver technique [14]. The IDMA system employed chip-interleaving process for user separation at the receiver side [14-17]. The authors in [14-17] aimed to improve the performance of the considered system in terms of system capacity or bit error rate with low complexity detection algorithm by using different interleaver codes and channel codes. The cooperative and dual-hop IDMA scheme was proposed in [1820]. In [18], a cooperative transmission system based on IDMA with DF and AF protocols for Rayleigh links was 
analysed. In [19], the authors focused on the partner selection strategies and outage probability expression in cooperative IDMA system in cellular network for superposition modulation. Bilim et al. developed a dual-hop IDMA system without the best relay selection over Weibull fading environments [20]. In [18-20], the authors examined the performance of the considered systems based on simulation and did not provide analytical expressions such as exact or approximated solutions. In this paper, a closed-form approximated CDF expression of a dual-hop DF IDMA system by using minimum mean square error (MMSE) detector is derived. The best relay selection method in dual-hop DF IDMA system is adopted for reducing the complexity of the system and the impact of best relay selection method on the outage performance of the dual-hop DF IDMA system is presented. To the best of the authors' knowledge, the proposed expression is the first approximated expression for a dual-hop DF IDMA network using best relay selection strategy in the literature that are based on the total SNR at the destination. Simulation results are verified the accuracy of the proposed analytical expression in the medium and high SNR region.

\section{System and Channel Model}

As shown in Fig. 1, we take into account a dual-hop relaying system based on DF IDMA system over Weibull links. We assume that there are $K$ cooperating users in the considered system and each user is willing to cooperate the other users by using DF protocol. In addition, each user is equipped with one antenna. The channel coefficients between source-relays or relays-destination are flat Weibull fading.

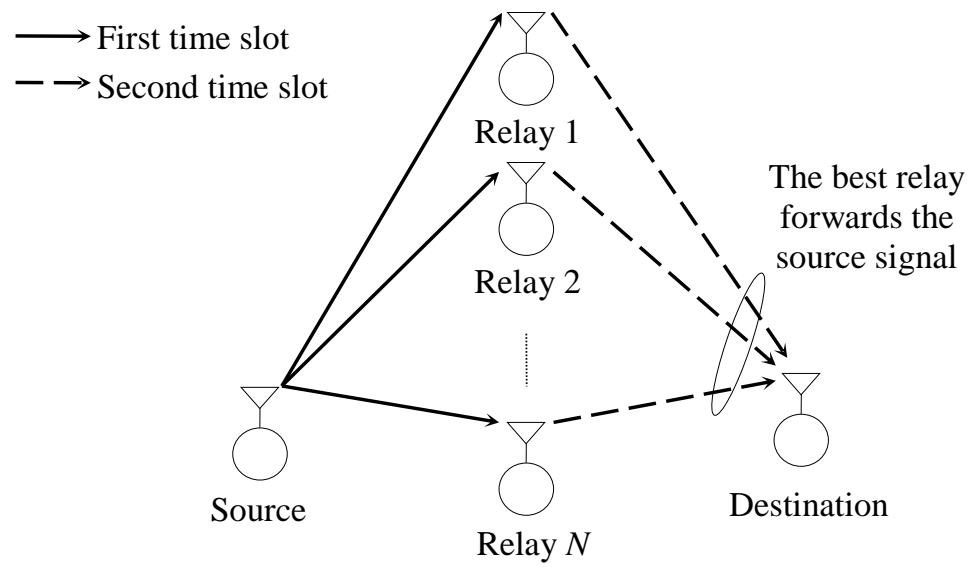

Fig. 1: Illustration of dual-hop DF IDMA for user $k$.

\subsection{Received signal models at relays and destination}

Signal transmission is operated in two time slots. Source information is transmitted to relays in the first time slot. Note that the source information is not sent to the destination due to considering dual-hop transmission model. Thus, the received signal at the $n$th relay node is expressed by

$$
y^{I}(t)=\sum_{j=0}^{J-1} \sum_{k=1, k \neq n}^{K} x_{k}(j) \pi_{k}\left(t-\tau_{s}-j T_{b}\right) h_{s r_{n}}+n^{I}(t)
$$

where $J$ is the frame length of the user $k, k=1,2, \ldots, K$ definitions number of cooperating users in the network. $x_{k}(j)$ is the $j$ th source symbol of the user $k . \pi_{k}(t)$ represents the combination of spreading and random interleaving sequences for user $k, T_{b}$ is the bit period, $\tau_{s}$ is the transmission delay. $h_{s r_{n}}$ denotes the Weibull coefficient between source and $n$th relay with $\Omega_{s r_{n}}=E\left(\left|h_{s r_{n}}\right|^{2}\right)=1$ and $c_{s r_{n}}$ is fading parameter. $n^{I}(t)$ is the additive white Gaussian noise (AWGN) with variance $\sigma_{n}^{2}=N_{0} / 2$ and zero mean. In the second time slot, relays which are cooperating with each other transmit the 
decoded source symbol to destination node by employing IDMA transmitter procedure. The total signal at the destination can be given as

$$
y^{I I}(t)=\sum_{j=0}^{J-1} \sum_{k=1}^{K} \sum_{n=1}^{N} \tilde{x}_{k}(j) \pi_{k}\left(t-D_{k, n}-\tau_{r_{n}}-j T_{b}\right) h_{r_{n} d}+n^{I I}(t)
$$

where $\tau_{s}=\tau_{r_{n}}, n^{I I}(t)$ and $D_{k, n}$ are AWGN and the transmit delay pending the second time slot, respectively. $h_{r_{n} d}$ is the Weibull coefficient between $n$th relay-destination channel. $N$ is the number of cooperative relays in the considered system. $\tilde{x}_{k}(j)$ is the decoded source symbol of user $k$ at relay nodes.

\subsection{Weibull fading model}

The PDF of instantaneous SNR, $\gamma_{i j}$, can be given according to Weibull distribution as

$$
p_{\gamma_{i j}}\left(\gamma_{i j}\right)=u_{i j}\left(\frac{\Gamma\left(\beta_{i j}\right)}{\bar{\gamma}_{i j}}\right)^{u_{i j}} \gamma_{i j}^{u_{i j}-1} \exp \left(-\left(\frac{\gamma_{i j} \Gamma\left(\beta_{i j}\right)}{\bar{\gamma}_{i j}}\right)\right.
$$

where $\bar{\gamma}_{i j}=E\left\langle h_{i j}^{2}\right\rangle E_{s} / N_{0} i, j \in\left\{s, r_{n}, d\right\}$ is the average SNR and $E\langle\cdot\rangle$ is the expectation operator. $u_{i j}=c_{i j} / 2$, $\beta_{i j}=1+2 / c_{i j}$ where $c_{i j}$ is Weibull fading parameter, $\Gamma(\cdot)$ is the Gamma function. $\gamma_{i j}$ is given by $\gamma_{i j}=\left|h_{i j}\right|^{2}\left(E_{s} / N_{0}\right)$.

\section{Outage Probability of Dual-Hop DF IDMA System with Best Relay Selection}

In this section, the outage probability of the considered system for Weibull channels is presented. For the outage probability, we have to present mutual information $\left(I_{i j}\right)$ between indirect link. The $I_{r_{n} d}$ between $n$th relay that is best relay and destination is written by

$$
I_{r_{n} d}=\frac{K}{2 L} \log \left(1+\frac{2 L \max _{n \in \alpha}\left(\gamma_{r_{n} d}\right)}{K^{2}[W]_{r_{n}, r_{n}}}\right)
$$

where $[W]_{r_{n}, r_{n}}$ is the MMSE detection element at relays and is defined by $[W]_{r_{n}, r_{n}}=\left[\left(R+\left(E_{s} / N_{0}\right)^{-1} I\right)^{-1}\right]_{r_{n}, r_{n}} . I$ is unit matrix and $L=\left(T_{b} / T_{c}\right)$ is the spreading gain where $T_{c}$ is chip period. $R$ is a cross correlation function between interleaved and spreading codes. When $I_{r_{n} d}$ falls to a certain specified rate of $\mathfrak{R}$, the outage probability occurs. The outage probability, when the best relay selection DF protocol is employed, can be written as

$$
P_{\text {out }}=\operatorname{Pr}\left[I_{r_{n} d}<\mathfrak{R}\right]=\operatorname{Pr}\left[\max _{n \in \alpha}\left(\gamma_{r_{n} d}\right)<\gamma_{t h-r_{n} d}\right\rfloor
$$

From (4) and (5), we have

$$
\gamma_{t h-r_{n} d}=2^{2 L \Re / K}-1 /\left(2 L / K^{2}[W]_{r_{n}, r_{n}}\right)
$$

where $\gamma_{t h-r_{n} d}$ is threshold value for the channel between the $n$th relay and destination. By substituting (6) into (5), the outage probability can be obtained as 


$$
P_{\text {out }}=\operatorname{Pr}\left[I_{r_{n} d}<\Re\right]=\operatorname{Pr}\left[\max _{n \in \alpha}\left(\gamma_{r_{n} d}\right)<2^{2 L \Re / K}-1 /\left(2 L / K^{2}[W]_{r_{n}, r_{n}}\right)\right]
$$

To find a closed-form outage probability expression of DF IDMA with best relay selection, we have to solve the outage probability expression in (7). However, the expression in (7) is very difficult. Using the method described in [21], we can bypass this difficulty and introduce a random variable $\varepsilon_{n}$ which denote the equivalent $\gamma_{i j}$ at the destination. This random variable, $\varepsilon_{n}$, take account of the links between source- $n$th relay and $n$th relay-destination. Therefore, $\varepsilon_{n}$ which is the PDF of the $s-r_{n}-d$ channel is expressed by

$$
p_{\varepsilon_{n}}(x)=\operatorname{Pr}\left(r_{n} \text { is off }\right) p_{\varepsilon_{n} \mid r_{n} \text { is off }}(x)+\operatorname{Pr}\left(r_{n} \text { is on }\right) p_{\varepsilon_{n} \mid r_{n} \text { is on }}(x)
$$

where $p_{\varepsilon_{n} \mid r_{n} \text { is off }}(x)$ is the conditional PDF and described by $p_{\varepsilon_{n}} \mid r_{n}$ is off $(x)=\delta(x)$ where $\delta(\cdot)$ is the Drac delta function. $\operatorname{Pr}\left(r_{n}\right.$ is off $)$ is the probability that the channel through $r_{n}$ is off and $\operatorname{Pr}\left(r_{n}\right.$ is off $)$ can be expressed as (9) by using [22, eq. (2.30)].

$$
\operatorname{Pr}\left(r_{n} \text { is off }\right)=1-\exp \left(-\left(\frac{\gamma_{t h-r_{n} d} \Gamma\left(\beta_{s r_{n}}\right)}{\bar{\gamma}_{s r_{n}}}\right)^{u_{s r_{n}}}\right)
$$

$\operatorname{Pr}\left(r_{n}\right.$ is on $)$ is the probability that the channel through $r_{n}$ is on and defined by $1-\operatorname{Pr}\left(r_{n}\right.$ is off $)$. By using (3), conditional PDF, $p_{\varepsilon_{n} \mid r_{n} \text { is on }}(x)$, can be given as

$$
p_{\varepsilon_{n} r_{n} \text { is on }}(x)=u_{r_{n} d}\left(\frac{\Gamma\left(\beta_{r_{n} d}\right)}{\bar{\gamma}_{r_{n} d}}\right)^{u_{r_{n} d}} x^{u_{r_{n} d}-1} \exp \left(-\left(\frac{x \Gamma\left(\beta_{r_{n} d}\right)}{\bar{\gamma}_{r_{n} d}}\right)^{u_{r_{n} d}}\right)
$$

Using (9), (10), the $\operatorname{Pr}\left(r_{n}\right.$ is on $)$ and $p_{\varepsilon_{n} \mid r_{n} \text { is off }}(x)$ definitions, (8) is rewritten as

$$
\begin{aligned}
p_{\varepsilon_{n}}(x)= & \left\{1-\exp \left(-\left(\frac{\gamma_{t h-r_{n} d} \Gamma\left(\beta_{s r_{n}}\right)}{\bar{\gamma}_{s r_{n}}}\right)^{u_{s r_{n}}}\right)\right\} \delta(x)+\left\{\exp \left(-\left(\frac{\gamma_{t h-r_{n} d} \Gamma\left(\beta_{s r_{n}}\right)}{\bar{\gamma}_{s r_{n}}}\right)^{u_{s r_{n}}}\right)\right\} \\
& \times u_{r_{n} d}\left(\frac{\Gamma\left(\beta_{r_{n} d}\right)}{\left.\bar{\gamma}_{r_{n} d}\right)}\right)^{u_{r_{n} d}} x^{u_{r_{n} d}-1} \exp \left(-\left(\frac{x \Gamma\left(\beta_{r_{n} d}\right)}{\bar{\gamma}_{r_{n} d}}\right)^{u_{r_{n} d}}\right)
\end{aligned}
$$

Eq. (11) denotes unconditional PDF of the instantaneous SNR for the $n$th $s-r_{n}-d$ link. According to $\varepsilon_{n}$, the outage probability of the $s-r_{n}-d$ link in (7) can be rewritten as

$$
P_{\text {total }}(x)=\operatorname{Pr}\left[\max _{n \in N}\left(\varepsilon_{n}\right)<x\right]=\prod_{n=1}^{N} \operatorname{Pr}\left[\varepsilon_{n}<x\right]=\prod_{n=1}^{N} P_{\varepsilon_{n}}(x)
$$

where $P_{\varepsilon_{n}}(x)$ is the CDF of $\varepsilon_{n}$ that can be obtained from (11) as 


$$
\begin{aligned}
P_{\varepsilon_{n}}(x) & =\int_{0}^{x}\left[\left\{1-\exp \left(-\left(\frac{\gamma_{t h-r_{n} d} \Gamma\left(\beta_{s r_{n}}\right)}{\bar{\gamma}_{s_{n}}}\right)\right\} \delta(x)\right.\right. \\
& +\left\{\exp \left(-\left(\frac{\gamma_{t h-r_{n} d} \Gamma\left(\beta_{s r_{n}}\right)}{\bar{\gamma}_{s r_{n}}}\right)^{u_{s_{n}}}\right)\right\} u_{r_{n} d}\left(\frac{\Gamma\left(\beta_{r_{n} d}\right)}{\bar{\gamma}_{r_{n} d}}\right)^{u_{r_{n} d}} x^{u_{r_{n} d}-1} \exp \left(-\left(\frac{x \Gamma\left(\beta_{r_{n} d}\right)}{\bar{\gamma}_{r_{n} d}}\right)\right] d x
\end{aligned}
$$

The integral in (13) is not analytically tractable due to the exponential term in second part of the integral depend on integral variable. Therefore, we simplify the computation of the integral in (13) by using the approximated PDF in [23, eq. (16)]. Hence, the approximated PDF of $\gamma_{i j}$ is given by

$$
p_{\gamma_{i j}}\left(\gamma_{i j}\right) \square u_{i j}\left(\frac{\Gamma\left(\beta_{i j}\right)}{\bar{\gamma}_{i j}}\right)^{u_{i j}} \gamma_{i j}^{u_{i j}-1}+\text { High OrderTerms }
$$

The detailed explanations about the approximated PDF in (14) can be found in [23]. By substituting (14) into (13), the CDF expression, $P_{\varepsilon_{n}}(x)$, follows

$$
\begin{aligned}
P_{\varepsilon_{n}}(x) & \square \int_{0}^{x}\left\{\left\{1-\exp \left(-\left(\frac{\gamma_{t h-r_{n} d} \Gamma\left(\beta_{s r_{n}}\right)}{\bar{\gamma}_{s r_{n}}}\right){ }^{u_{s r_{n}}}\right)\right\} \delta(x)\right. \\
+ & \left.\left.\exp \left(-\left(\frac{\gamma_{t h-r_{n} d} \Gamma\left(\beta_{s r_{n}}\right)}{\bar{\gamma}_{s r_{n}}}\right)^{u_{s r_{n}}}\right)\right\} u_{r_{n} d}\left(\frac{\Gamma\left(\beta_{r_{n} d}\right)}{\bar{\gamma}_{r_{n} d}}\right)^{u_{r_{n} d}} x^{u_{r_{n} d}-1}+\text { H.O.T. }\right] d x
\end{aligned}
$$

After some mathematical manipulations, $P_{\varepsilon_{n}}(x)$ is derived as

$$
P_{\varepsilon_{n}}(x) \square\left\{1-\exp \left(-\left(\frac{\gamma_{t h-r_{n} d} \Gamma\left(\beta_{s r_{n}}\right)}{\bar{\gamma}_{s r_{n}}}\right)^{u_{s s_{n}}}\right)\right\}+\left\{\exp \left(-\left(\frac{\gamma_{t h-r_{n} d} \Gamma\left(\beta_{s r_{n}}\right)}{\bar{\gamma}_{s r_{n}}}\right)^{u_{s r_{n}}}\right)\right\}\left\{\left(\frac{\Gamma\left(\beta_{r_{n} d}\right)}{\bar{\gamma}_{r_{n} d}}\right)^{u_{r_{n} d}} x^{u_{r_{n} d}}\right.
$$

The derived expression in (16) is substituted into (12) and then, the total CDF of the system is obtained as

$$
P_{\text {total }}(x) \square \prod_{n=1}^{N}\left\lfloor\left\{1-\exp \left(-\left(\frac{\gamma_{t h-r_{n} d} \Gamma\left(\beta_{s r_{n}}\right)}{\bar{\gamma}_{s r_{n}}}\right)^{u_{s r_{n}}}\right)\right\}+\left\{\exp \left(-\left(\frac{\gamma_{t h-r_{n} d} \Gamma\left(\beta_{s r_{n}}\right)}{\bar{\gamma}_{s r_{n}}}\right)^{u_{s r_{n}}}\right)\right\}\left\{\left(\frac{\Gamma\left(\beta_{r_{n} d}\right)}{\bar{\gamma}_{r_{n} d}}\right)^{u_{r_{n} d}} x^{u_{r_{n} d}}\right\rfloor\right.
$$

In order to find outage probability, we have to employ the threshold value, $\gamma_{t h-r_{n} d}$, in the proposed expression given in (17). 


\section{Numerical Examples}

The results obtained using the proposed expression of dual-hop DF IDMA system with best relay selection scheme are presented in this section. Figs. 2 and 3 illustrate the outage probability of the considered system versus the average SNR for different Weibull parameters and different number of relays. In these example sets, we assume that the values of the cooperating user, spreading gain and specified rate $(K, L, \Re)$ in eq. $(6)$ are set to $(16,16,1)$. These figures show excellent agreement between the analytical results and simulation results especially from medium to high values of SNR. Significant observations can be reported from Figs. 2 and 3. As expected, the outage probability of the considered system is improved while both the fading parameter of the indirect links and the number of relay increase. As an example, it can be seen from Fig. 2 that an outage performance of $10^{-2}$ happens at $\mathrm{SNR} \approx 33 \mathrm{~dB}$ for $c_{r_{n} d}=1.0$, while it occurs at $\mathrm{SNR} \approx 21 \mathrm{~dB}$ and $\mathrm{SNR} \approx 17 \mathrm{~dB}$ for fading links with $c_{r_{n} d}=2.0$ and $c_{r_{n} d}=3.0$, respectively. It can be noticed from the curves in Fig. 2 that indirect links are affected by the fading parameter $c_{r_{n} d}$.

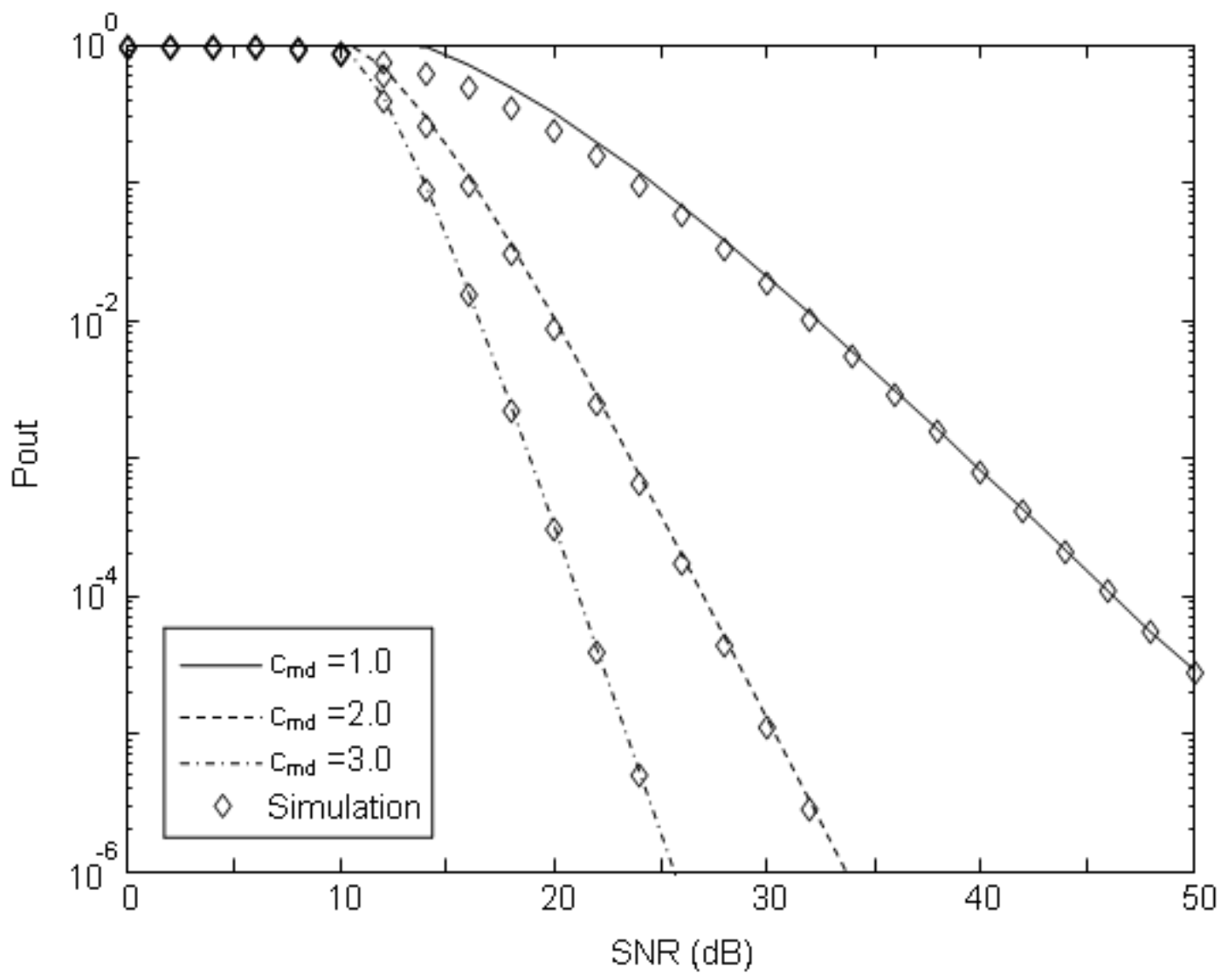

Fig. 2: Outage probability of DF IDMA with best relay selection for different Weibull fading parameter for $N=2$.

In Fig. 3, we show the impact of the number of relays in the best relay selection on the outage probability of the dual-hop DF IDMA system with $c_{r_{n} d}=2.0$. The results in this figure show that the analytical results obtained by the proposed approximated expression are match well with simulation results. Different number of relays are considered, $N=1,2,3$ and 4 as well for good comparison. It is observed that the outage probability is improved with increasing the number of relays in this figure, as expected. As an example, an outage probability of $10^{-1}$ occurs at $\mathrm{SNR} \approx 24 \mathrm{~dB}$, $\mathrm{SNR} \approx 19 \mathrm{~dB}, \mathrm{SNR} \approx 16 \mathrm{~dB}$ and $\mathrm{SNR} \approx 15 \mathrm{~dB}$ for no selection, $N=2,3$ and 4 , respectively. 


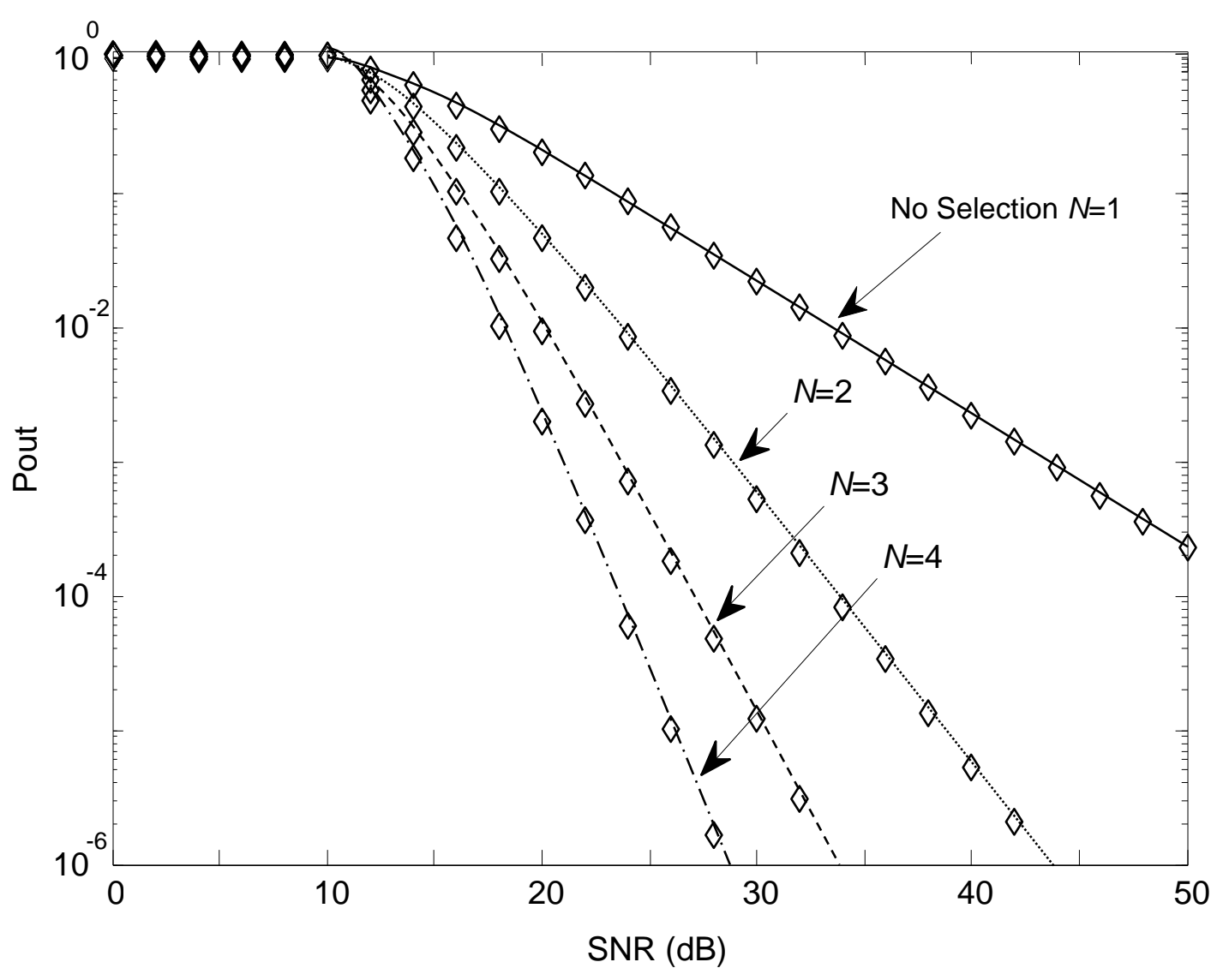

Fig. 3: Outage probability of DF IDMA with best relay selection in case of $c_{r_{n} d}=2.0$ with different number of relays.

\section{Conclusion}

A new analytical expression for the CDF of a two-hop DF IDMA network with best relay selection has been derived. The proposed expression was employed to achieve the results for the outage performance of the considered wireless networks over Weibull environments. The impact of increasing the number of available users (relays) and fading parameter on the outage performance was investigated. It was also observed that simulation results verified the tightness of the derived expression at medium and high SNR values.

\section{Acknowledgements}

This work was supported by the Scientific and Technological Research Council of Turkey (TUBITAK) under project grant 114E242. The authors thank to the Scientific Research Projects Coordinating Office of Erciyes University (EU-BAP, Project No: FDK-2014-5159).

\section{References}

[1] A. Bletsas, H. Shin, M. Z. Win and A. Lippman, "A simple Cooperative diversity method based on network path selection,” IEEE J. Select. Areas Commun., vol. 24, no. 3, pp. 659-672, 2006.

[2] J. N. Laneman, N. C. David and G. W. Wornell, "Cooperative diversity in wireless networks: efficient protocols and outage behavior," IEEE Trans. Inf. Theory, vol. 50, no. 12, pp. 3062-3080, 2004.

[3] J. N. Laneman, G. W. Wornell and D. N. C. Tse, "An efficient protocol for realizing cooperative diversity in wireless networks," in Proceedings of IEEE ISIT01, Washington DC, June 2001, p. 294.

[4] A. Sendonaris, E. Erkip and B. Aazhang, "User cooperation diversity, Part I: system description," IEEE Trans. Commun., vol. 51, no. 11, pp. 1927-1938, 2003. 
[5] A. Sendonaris, E. Erkip, and B. Aazhang, "User cooperation diversity, Part II: implementation aspects and performance analysis," IEEE Trans. Commun., vol. 51, no. 11, pp. 1939-1948, 2003.

[6] S. S. Ikki and M. H. Ahmed, "Performance analysis of adaptive decodeand-forward cooperative diversity networks with the best relay selection scheme," IEEE Trans. Commun., vol. 58, no. 1, pp. 68-72, 2010.

[7] S. S. Ikki and M. H. Ahmed, "Exact error probability and channel capacity of the best-relay cooperative-diversity networks," IEEE Signal Process. Lett., vol. 16, no. 12, pp. 1051-1054, 2009

[8] S. S. Ikki and M. H. Ahmed, "On the performance of cooperative-diversity networks with the Nth best-relay selection scheme," IEEE Trans. Commun., vol. 58, no. 11, pp. 3062-3069, 2010.

[9] Q. Shi and Y. Karasawa, "Error probability of opportunistic decodeand-forward relaying in Nakagami- $m$ fading channels with arbitrary m," IEEE Wireless Commun. Lett., vol. 2, no. 1, pp. 86-89, 2013.

[10] H. Guo and J. Ge, "Performance analysis of two-way opportunistic relaying over Nakagami- $m$ fading channels," Electron. Lett., vol. 47, no. 2, pp. 150-152, 2011.

[11] S. S. Soliman and N. C. Beaulieu, "Exact analysis of dual-hop AF maximum end-to-end SNR relay selection," IEEE Trans. Commun., vol. 60, no. 8, pp. 2135-2145, 2012.

[12] G. Li, J. Chen, Y. Huang, and G. Ren. "Outage performance of multiple-input-multiple-output decode-and-forward relay networks with the $N$ th-best relay selection scheme in the presence of co-channel interference," IET Commun., vol. 8, no. 15, pp. 2762-2773, 2014.

[13] N. Suraweera and N. C. Beaulieu, "Optimum combining in dual-hop AF relaying for maximum spectral efficiency in the presence of co-channel interference," IEEE Trans. Commun., vol. 63, no. 6, pp. 2071-2080, 2015.

[14] L. Ping, L. Liu, K. Wu and W. K. Leung, "Interleave-division multiple access," IEEE Trans. Wireless Commun., vol. 5, no. 4, pp. 938-947, 2006.

[15] K. Kusume, G. Bauch and W. Utschick, "IDMA vs. CDMA: analysis and comparison of two multiple access schemes," IEEE Trans. Wireless Commun., vol. 11, no. 1, pp. 78-87, 2012.

[16] M. Bilim, N. Kapucu and I. Develi, "A new approach to random interleavers for traditional IDMA systems," in Proceedings of IEEE Symposium on Computers and Communications, vol. 17, Cappadocia, July, 2012, pp. 209-212.

[17] M. Bilim, N. Kapucu and I. Develi, "Effect of repetition coding on the BER performance of interleave-division multiple access systems," Turkish Journal of Electrical Engineering \& Computer Sciences, vol. 24, no. 2, pp. 1412$1420,2016$.

[18] Z. Luo, D. Gurkan, Z. Zan, A. K-S. Wong and S. Oiu, "Cooperative communication based on IDMA," in Proceedings of the $5^{\text {th }}$ International Conference on Wireless Communications, Networking and Mobile Computing, Sep. 2009, pp. $1-4$.

[19] X. Wang, H. Zhang and D. Li, "Partner selection based on IDMA superposition modulation in cooperative cellular Networks," in Proceedings of the Vehicular Technology Conference, May 2010, pp. 1-4.

[20] M. Bilim, N. Kapucu and I. Develi, "Dual-Hop N-Relay Assisted Transmission for IDMA Systems over Weibull Fading Channels," Elektronika IR Elektrotechnika, vol. 21, no. 4, pp. 69-72, 2015.

[21] N. C. Beaulieu and J. Hu, "A closed-form expression for the outage probability of decode-and-forward relaying in dissimilar Rayleigh fading channels," IEEE Commun. Lett., vol. 10, no. 12, pp. 813-815, 2006.

[22] M. K. Simon and M.-S. Alouini, Digital Communication Over Fading Channels, $2^{\text {nd }}$ ed. Hoboken, NJ, USA: Wiley, 2005.

[23] S. Ikki and S. Aïssa, "Performance analysis of amplify-and-forward relaying over Weibull-fading channels with multiple antennas," IET Commun., vol. 6, no. 2, pp. 165-171, 2012. 